\title{
Thyroid Cancer Cells Boundary Location by a Fuzzy Edge Detection Method
}

\author{
C.C. Leung, F.H.Y. Chan, and \\ K.Y. Lam \\ The University of Hong Kong \\ Pokfulam, Hong Kong. \\ ccleung@eee.hku.hk \\ fhychan@eee.hku.hk \\ akylam@hkucc.hku.hk
}

\author{
P.C.K. Kwok \\ The Open University \\ of Hong Kong \\ Hon Min Tin, Hong Kong. \\ ckkwok@ouhk.edu.hk
}

\author{
W.F. Chen \\ First Military \\ Medical University \\ Guangzhou, China. \\ chenwf@fimmu.edu.cn
}

\begin{abstract}
Morphometric assessment of tumor cells is important in the prediction of biological behavior of thyroid cancer. In order to automate the process, the computer-based system has to recognize the boundary of the cells. Many methods for the boundary detection have appeared in the literature and some of them applied to microscopic slice analysis. However, there is no reliable method since the gray-levels in the nuclei are uneven and are similar to the background. In this paper, a Fuzzy Edge Detection Method is used and is based on an improved Generalized Fuzzy Operator. The method enhances the nuclei and effectively separates the cells from the background.
\end{abstract}

\section{Introduction}

Morphometric assessment of the different parameters of tumor cells is important in the study of thyroid cancer. However, objective measurement is not possible without the help of reliable computer software. The objective assessment includes some algorithms [1-5] to detect the boundary of the cells based on the gradient method. However, these conventional edge detection methods cannot detect the boundary with reasonable accuracy. The reasons are (1) for the incomplete cancer cells, the gray-level of its nucleus is lighter than the complete cancer cells; (2) the nucleus and the background have similar gray levels; (3) in some cells, the nucleus and the cell membrane are very distinct that they may be separated into two concentric rings and double counted. These problems are evident in a conventional thresholding algorithm such as the Yanowitz et al [8] method. Adaptive thresholding proposed by Chan et al [7] shows some improvement. It is a region based heuristic algorithm with the following steps.

1. Image smoothing using average filtering.
2. Obtain the gray-level gradient magnitude.

3. Derive the threshold surface by deforming the original image gray-level surface.

4. The threshold surface interpolation.

5. Segmentation based on the threshold surface.

However, incomplete cells are still not detected reliably with this method. Double counting is sometimes occurred.

In this paper, a Fuzzy Edge Detection Method is proposed. It is based on the Generalized Fuzzy Operator (GFO) [6]. It enhances those cells whose gray-level is similar to the background and produces complete boundary of the cells.

\section{Definitions of GFO}

Definition 1. Denote the Generalized Fuzzy Set (GFS) $S$ in the region $R$ as

$$
S=\int \frac{\mu_{S}(x)}{x}, x \in R
$$

Where $\mu_{S}(x) \in[-1,1]$ is called the Generalized Membership Function (GMF) of $S$ on $R$. Since $\mu_{S}(x) \in[-1,0)$. The GMF of $x$ in $S$ is not a subordinate on $\mathrm{R}$, for $\mu_{S}(x) \in[0,1]$. The GFM of $x$ in $S$ is subordinate on $R$; and $\mu_{S}(x)=0$. The fuzzy bound point function (FBF) in $S$ is on $R$.

According to the definition in [6], we can write,

$\mu_{\mathrm{T}}(x)=\mathrm{GFO}[\mu \mathrm{s}(x)]=$ 


$$
\left\{\begin{array}{cc}
\sqrt[\beta]{1-\left[1+\mu_{s}(x)\right]^{\beta}}, & -1 \leq \mu_{s}<0 \\
{\left[\mu_{s}(x)\right]^{\beta},} & 0 \leq \mu_{s}<r \\
\sqrt[\beta]{1-\alpha\left[1-\mu_{s}(x)\right]^{\beta}}, & r \leq \mu_{s}(x) \leq 1
\end{array}\right.
$$

From (2-2), we can deduce the following properties:

Property 1. When $\beta \rightarrow \infty$,

$\mu_{T}(x)=\left\{\begin{array}{cc}1, & -1 \leq \mu_{s}(x)<0 \\ 0, & 0 \leq \mu_{s}(x)<r \\ 1, & r \leq \mu_{s}(x) \leq 1\end{array}\right.$

Property 2. When $\beta>1$,

$$
\left\{\begin{array}{r}
\mu_{T}(x)>\mu_{S}(x), \text { if }-1 \leq \mu_{S}(x)<0 \\
r<\mu_{S}(x) \leq 1 \\
\mu_{T}(x)<\mu_{S}(x), \text { if } 0<\mu_{S}(x) \leq r
\end{array}\right.
$$

Then the generalized fuzzy set $S$ becomes a normal fuzzy set $T$, such that

$$
T=\mathrm{GFO}[S]
$$

\section{Enhancement and Edge detection}

From Section 2, we use the GFO to map all the pixels in the original image into the GFS. For an image $X(i, j)$, where $i=1,2, \ldots, \mathrm{N}$ and $j=1,2, \ldots, \mathrm{M}$. $P(i, j)$ :

We use a Sine function to map $X(i, j)$ into a fuzzy set

$$
P(i, j)=\sin \left\{\frac{\pi}{2}\left(1-\frac{X(i, j)-X_{\min }}{D}\right)\right\}
$$

where $\frac{X_{\max }-X_{\min }}{2} \leq D$.

Then, $P \in[-1,1]$ is mapped to the new fuzzy set $P^{\prime}$ as shown in the following equation,

$$
P^{\prime}(i, j)=\left\{\begin{array}{cc}
\sqrt[\beta]{\left(1-(1+P(i, j))^{\beta}\right.}, & -1 \leq P<\gamma-\Delta \\
P^{2}(i, j), & \gamma-\Delta \leq P \leq \gamma+\Delta \\
\sqrt[\beta]{\left(1-\alpha(1-P(i, j))^{\beta}\right.}, & \gamma+\Delta<P \leq 1
\end{array}\right.
$$

By setting $\beta=2$, and the newer image $X^{\prime}(i, j)$ is

$$
X^{\prime}(i, j)=X_{\min }+D\left\{1-\left[\frac{\sin ^{-1}\left(P^{\prime}(i, j)\right)}{\frac{\pi}{2}}\right]\right\}
$$

In the present application, the objective of Morphometric assessment is to locate the boundary of the thyroid cancer cell. We used the improved GFO to put the boundary gray level be mapped to a distinctly different gray levels. Now, the membrane's boundary has a gray level distribution in the range $[\gamma-\Delta \gamma+\Delta]$. In equation (3-2), this range is mapped to a minimum value in $P^{\prime}$. Above or below this range, it is mapped to progressively higher values as shown in Figure 1. The characteristics of the improved GFO is a "V" shaped curve located in [-1 1 ] of $P$. This function resembles a band-pass function so that the edge pixels are mapped to a range of values distinct from the others. The parameter " $\gamma$ " can be adjusted to shift the "V" type function along the $P(i, j)$ axis, depending on the actual gray levels of the boundary of interest. $\alpha$ can affect the right arm of the " $V "$ shaped function.

\section{Results and Discussions}

We implemented our method in a Pentium PC. The algorithm was programmed in the MATLAB. The original image of the thyroid cancer cell is shown in figure 2 . Point " $A$ " is an incomplete cell with a hollow-looking nucleus. Point " $B$ " shows another incomplete cell, so that the graylevel of this cell is almost the same as the background. Moreover, some cells overlap and merge together. Figure 3 is a binary image that is the result of using the Adaptive Thresholding Method [7]. In using this method, an area with solid interior is counted as a cell. However, in point " $A$ ", the hollow ring will not be counted as a cell since certain tissue boundary is also processed as the hollow ring in the same slice shown in point " $A$ ". In point " $B$ ", an irregular pattern shows up in the result and it will not be counted as a cell. It is caused by no function to enhance the incomplete cell in this method. Figure 4 is the result based on the new proposed method. The boundaries show up distinctly in both points " $A$ " and " $B$ ". In another case, when two cells appeared to be merged together, they are separated after processing.

In the example, $\gamma, \alpha, \Delta$ and $\mathrm{D}$ are chosen as -0.9946 , 
$0.1,0.01$ and 79.406 respectively for the present example. The characteristics of the fuzzy set are shown in Figure 5. The relationship between the original and the new pixel values is shown in Figure 6. At an original gray level of around 160 , the pixels are mapped to a minimum value. The other pixels will be mapped to other distinctly different gray levels. In this example, the thyroid cancer cells have boundary pixels with gray levels of around 160. For the incomplete cells, the boundaries are enhanced.

\section{Conclusion}

The proposed Fuzzy algorithm is a simple, effective and efficient method for edge detection and enhancement. We have demonstrated that it is very suitable for application in the counting of thyroid cancer cells captured from a microscopic slice. It solves the problems better than the Adaptive Thresholding Method.

\section{References}

[1] Nevatia, R., "A color edge detection and its use in scene segmentation". IEEE Trans. Syst. Man and Cybern., SMC-7, vol.11, pp.820-825, 1977.

[2] Shen, J., "An optimal linear operator for edge detection". Proc. of IEEE Computer Vision and Pattern Recognition Conference, pp.109-114, 1986.

[3] Rosin, P.L. and West, G.A.W., "Segmentation of Edges into Lines and Arcs". Image Vision Computing, vol.7, pp.109-114, 1989.

[4] R.F. Wagner, M.F. Insana, and S.W. Smith, "Fundamental correction lengths of coherent speckle in medical ultrasonic images". IEEE Trans. Ultrason., Ferroelect., Freq. Contr., vol. 35, pp.34-44, 1988.

[5] A.C. Bovik, "On detecting edges in speckle imagery". IEEE Trans. Signal Processing, vol. 36, pp.1618-1627, 1988.

[6] Chen, W.F., Lu, X.Q., Chen, J.J., and Wu, G.X., "A new algorithm of edge detection for color image: Generalized fuzzy operator". Science in China (Series A), vol.38, No.10, pp.1272-1280, 1995.

[7] Chan, F.H.Y., Lam, F.K., and Zhu, H., "Adaptive Thresholding by Variational Method". IEEE Trans. Image Processing, Vol.7, No.3, pp.468-473, 1998.

[8] Yanowitz, S.D, and Bruckstein A.M., "A new method for image segmentation". Comput. Vision, Graph., Image Process., Vol.46, pp.82-95, 1989.

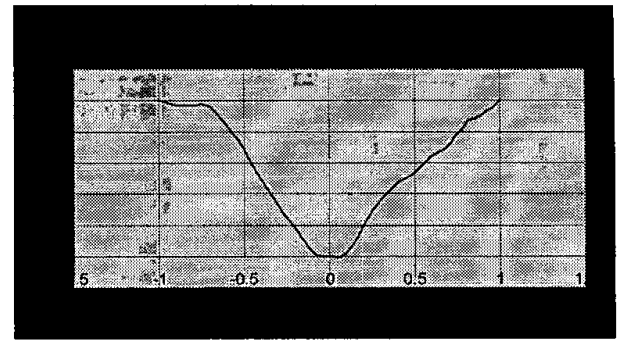

Figure 1. The characteristics of the GFO $(\gamma=0), \alpha$ and $\gamma$ can change the shape based on the " $\mathrm{V}$ " shaped curve.

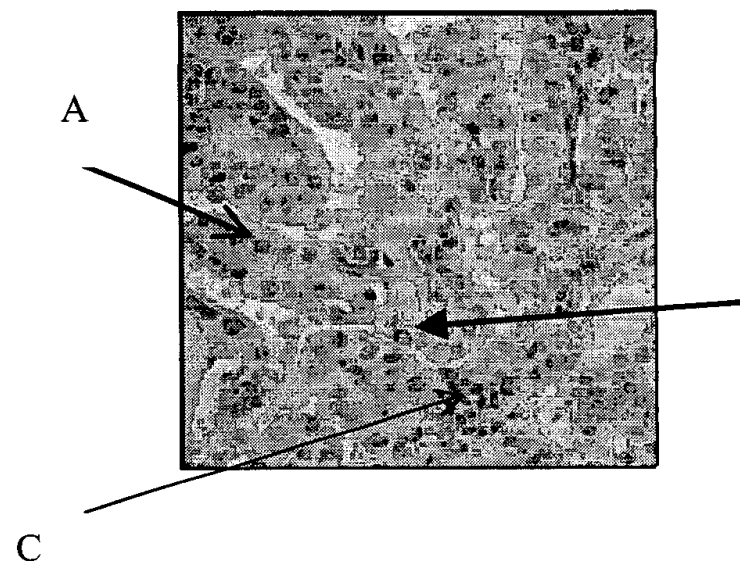

B

Figure 2 The microscopic slice contains two types of thyroid cancer cells, one is in growing so that it's nuclei is not in rigid (point A). However, some cells its nucleus is rigid but lighter (point $\mathrm{B}$ ). The other is the completed growth cell (point $\mathrm{C}$ ), the nucleus is rigid and color is black. 


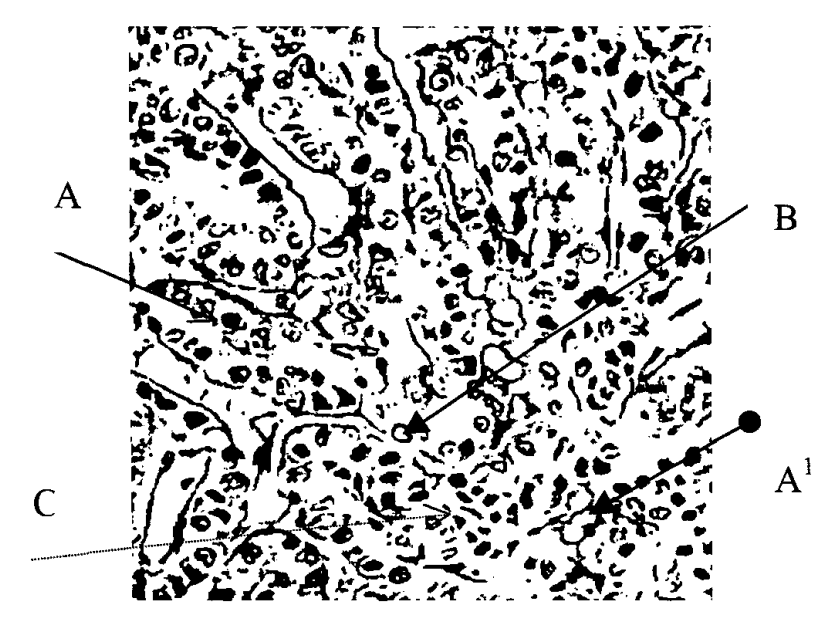

Figure 3. Using the Adaptive thresholding method to detect the thyroid cancer cells. Some of the cells cannot be counted.

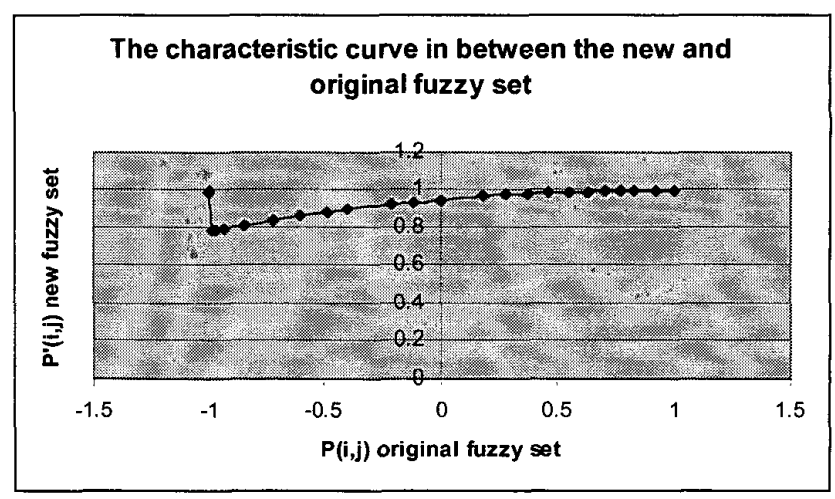

Figure 5. The characteristics of the GFO are used in the microscopic slice. $\gamma$ is set on -0.9946 .

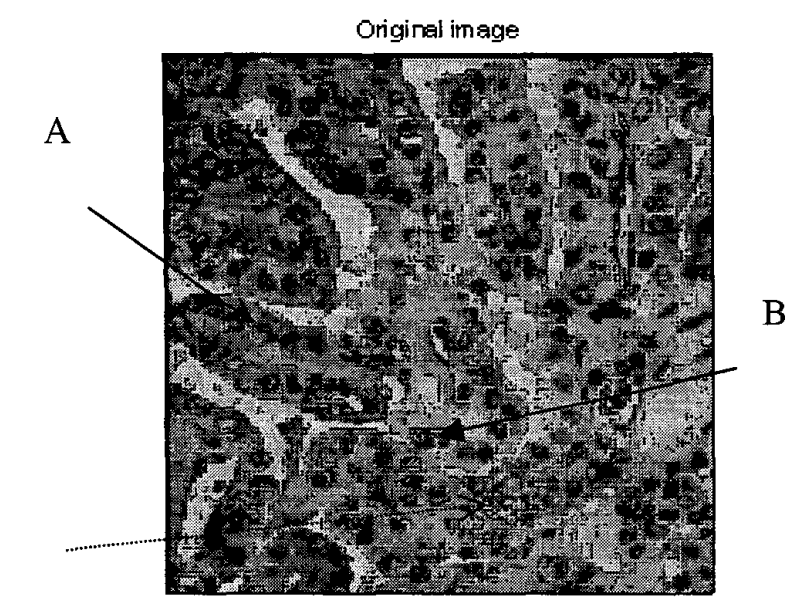

Figure 4. Using the improved GFO to process the image, most of the cells can be detected. The problem occurs in the result of Adaptive Thresholding method can be solved.

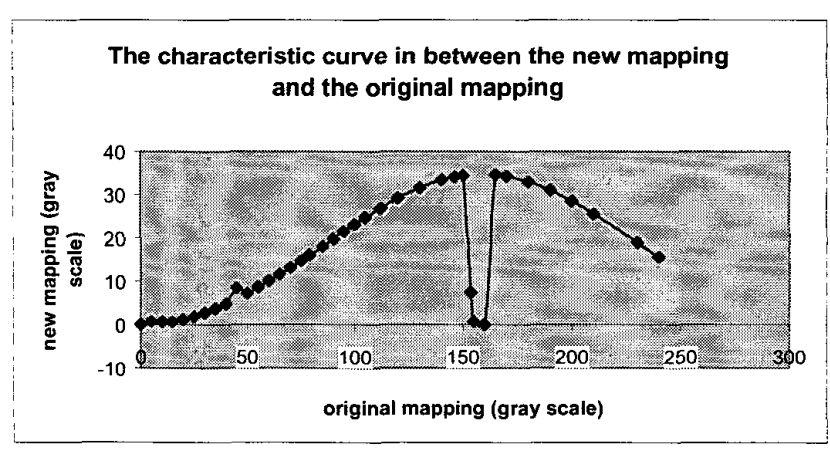

Figure 6. The relationship of the original image and the GFO image. The gray scale nearly 160 can be enhanced in the new image. 